\title{
A Divide-and-Conquer Approach to Estimating Minimum/Maximum Leakage Current
}

\author{
Guang-Wan Liao \\ Graduate Institute of Electronics Engineering \\ National Taiwan University \\ Taipei, Taiwan
}

\author{
Ja-Shong Feng and Rung-Bin Lin \\ Department of Computer Science and Engineering \\ Yuan Ze University \\ Chung-Li, Taiwan \\ Email: csrlin@cs.yzu.edu.tw
}

\begin{abstract}
Forcing primary inputs and memory elements into certain logic values is a viable method to lower standby leakage current for CMOS circuits. This paper presents a divide-andconquer approach to finding out an input vector that leads a circuit into a low (high) leakage state. With only a small fraction of time, our approach can achieve up to $11 \%$ smaller leakage than the lowest and up to $20 \%$ larger leakage than the highest respectively attainable with $100 \mathrm{~K}$ random vectors for the large ISCAS benchmark circuits. Our results are as good as that obtained by simulated annealing and genetic algorithm that run for a very long time. Due to its divide-and-conquer nature, it will be scalable for even larger circuits.
\end{abstract}

\section{INTRODUCTION}

Leakage power increasingly dominates the total power consumption of a circuit as process technologies aim to boost circuit speed by lowering the threshold voltage and thinning device gate oxide [1-4]. Numerous methods have been developed to harness this problem [5]. Dual (multiple) threshold voltage technique enables us to use low-threshold devices on critical paths to shorten path delay and high-threshold devices on non-critical paths to curtail leakage power [6-7]. Dynamically or statically applying body-bias to a certain logic block can also increase device threshold voltage in that block [8-10]. Transistor stacking which adds a controlled transistor along the current charging/discharging paths is used to curtail sub-threshold leakage current in standby mode [1113]. Input vector control that sets the logic values of primary inputs, latches, and flip-flops into known logic values in standby mode can be used to drive internal gates into a state maximizing the stacking effect [14-21]. Circuit techniques [22-25] are proposed to lower the gate leakage. Input-vector control combined with pin-reordering technique [26] is employed to reduce the gate leakage. Combinations of the above methods usually can achieve more leakage power reduction.

It is well known that the leakage current of a circuit is a function of its input logic value. Finding an input vector that leads a circuit into its highest or lowest leakage state is an active research area. Finding an input vector to place a circuit at its lowest leakage state is an NP-complete problem. The minimum leakage input vector can be used to reduce the leakage current in the standby mode, whereas the maximum leakage (input vector) can be used as a reference for IDDQ testing and maximum power estimation. Halter and Najm first proposed the idea of using natural stacking to lower the standby leakage current [14]. They used a statistical approach to deciding the number of input vectors needed to achieve a certain confidence level. Chen, Johnson, Wei, and Roy showed that the vector found by a genetic algorithm can outperform the vector selected from 50,000 randomly sampled vectors [11]. Johnson, Somasekhar, and Roy proposed a heuristic based on leakage observability defined for each input [12]. Naidu and Jcobs formulated the problem of finding the lowest leakage state into an integer linear programming model [16]. This model is then relaxed into an LP model and solved by lp_solve with randomized rounding. Aloul Hassoun, Sakallah, and Blaauw[17] transformed this problem into a Boolean satisfiability problem which is solved by a SAT solver. Abdollahi, Fallah, and Pedram [18] transformed this problem into a leakage computing network that serves as an input to a SAT solver for computing the total leakage of a circuit. Bobba and Hajj [19] proposed to use the circuit structure and the logic functionality to create a constraint graph on which a heuristic is developed to find the maximum leakage current. Ferre and Figueras [20] modeled the lowest-leakage-state finding problem as a testing problem. A circuit is first partitioned into many subcircuits before ATPG is used to find a vector. Note that a method used to find the lowest leakage vector can also be used to find the highest leakage vector or vice versus. Chopra and Vrudhula [21] proposed to perform a pseudo Boolean enumeration on an integer valued decision diagram. To handle a circuit with a large number of inputs, the circuit represented as hypergraphs are partitioned into small parts. All the above methods make a considerable progress in solving this problem, but none of them shows its capability of handling large circuits.

In this paper we present a divide-and-conquer method to find out a better low/high leakage state for large circuits. Both subthreshold and gate leakage are considered. Our key idea is to cluster closely correlated inputs into a number of disjoint groups, each of which contains a dozen of inputs such that exhaustive search for the lowest/highest leakage state can be efficiently performed for these inputs. The grouping approach is different from that presented in $[20,21]$ where logic gates are put into groups, but here we put the primary inputs of a circuit into groups. Gray code is employed to accelerate the exhaustive search. With only a small fraction of time, our approach can achieve up to $11 \%$ smaller leakage than the lowest and up to $20 \%$ larger leakage than the highest respectively attainable with $100 \mathrm{~K}$ random vectors for the large ISCAS benchmark circuits. Our results are as good as that obtained by simulated annealing and genetic algorithms that run for a very long time. The time complexity of our algorithm is $\mathrm{O}\left(m 2^{n / m}\right)$ where $n$ is the number of inputs and $m$ is the number of groups. As shown by the experimental results, our approach is scalable for large circuits. 
The rest of this paper is organized as follows. Section II studies leakage computation for basic cells and more complex circuits. Section III delineates our divide-and conquer approach. Section IV presents our findings. The last section draws some conclusions.

\section{LEAKAGE COMPUTATION}

Although there exists many leakage paths in a device, subthreshold leakage now and gate leakage in the future are the major leakage sources. Leakage current can be obtained using SPICE simulation as well as some analytical formulas $[11,27]$. In this paper, we will use SPICE to obtain the leakage current of a basic CMOS cell using BSIM4 [27]. Fig. 1 gives a circuit example to show how we define the subthreshold leakage $I_{s}$ and the gate leakage $I_{g}$. Let $Q$ denote the set of inputs with logic value 0 . Then, $I_{s}=I_{v}-\sum_{i \in Q}\left|I_{i}\right|$ is defined as the total current $I_{v}$ drawn from the power source minus those currents flowing out the gate terminals. $I_{g}=\left|I_{A}\right|+\left|I_{B}\right|+\left|I_{C}\right|$ is defined as the total amount of current entering or getting out from the gate terminals. Then, the total leakage is $I_{t}=I_{s}+I_{g}$.

With the prevalent use of standard cell designs, a design consists of many basic logic cells. A look-up table (LUT) with precharacterized leakage current is used to facilitate leakage power estimation of a more complex circuit. However, due to the complication of gate leakage, we suspect whether the leakage current of a more complex circuit can be simply an algebraic sum of the leakage current of individual cells. The following experiment is performed to validate this issue. Fig. 2 shows an example of an inverter chain. The circuit with the primary input tied to ground is given in Fig. 2(A). When LUT-based model is used to compute the leakage current, the circuit is decomposed into the one shown in Fig. 2(B). Ideally, $V_{A}=1.8 \mathrm{~V}$ and $V_{B}=0 \mathrm{~V}$ when $V_{D D}=1.8 \mathrm{~V}$. However, when the circuit given in Fig. 2(A) is put into its steady state, we have $V_{A}=1.7934 \mathrm{~V}$ and $V_{B}=0.0024 \mathrm{~V}$. When a transistor turns on, a voltage drop across the on-resistance of the transistor appears if there is leakage current on it. This is the reason why $V_{A}$ and $V_{B}$ in the circuit given in Fig. 2(A) are not in their ideal voltage value. This incurs some error in the total leakage current calculation, but the error is too small to affect the accuracy. As it can be observed from TABLE I that the difference between the leakage obtained by LUT-based model and that by SPICE simulation is within $1 \%$ for a full adder. Our conclusion is that the whole circuit leakage current can be obtained by summing the leakage currents of individual gates for the circuits composed of basic CMOS gates.

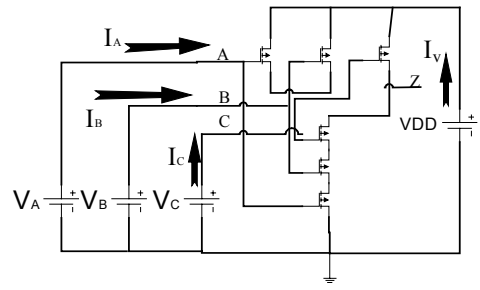

Figure 1. An example circuit for leakage computation

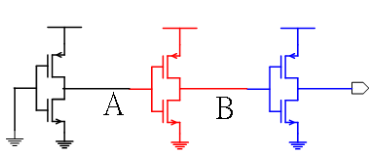

(A)

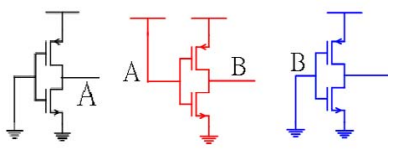

(B)
Figure 2. An example circuit for leakage computation
TABLE I. LEAKAGE CURRENT FOR A FULL ADDER

\begin{tabular}{|c|c|c|c|c|c|}
\hline \multicolumn{3}{|c|}{ Input } & \multirow{2}{*}{$\begin{array}{c}\text { SPICE } \\
(\mathrm{nA})\end{array}$} & \multirow{2}{*}{$\begin{array}{l}\text { LUT } \\
\text { (nA) }\end{array}$} & \multirow{2}{*}{$\frac{L U T-S P I C E}{L U T}$} \\
\hline A & B & Cin & & & \\
\hline 0 & 0 & 0 & 1.05308 & 1.05410 & $0.10 \%$ \\
\hline 0 & 1 & 0 & 1.16706 & 1.16850 & $0.12 \%$ \\
\hline 1 & 0 & 0 & 1.16700 & 1.16850 & $0.13 \%$ \\
\hline 1 & 1 & 0 & 1.06168 & 1.06240 & $0.07 \%$ \\
\hline 0 & 0 & 1 & 0.79267 & 0.79331 & $0.08 \%$ \\
\hline 0 & 1 & 1 & 0.95389 & 0.95444 & $0.06 \%$ \\
\hline 1 & $\begin{array}{l}0 \\
1\end{array}$ & & $\begin{array}{l}0.95386 \\
0.80127\end{array}$ & $\begin{array}{l}0.95384 \\
0.80158\end{array}$ & $\begin{array}{l}0.00 \% \\
0.04 \%\end{array}$ \\
\hline
\end{tabular}

\section{DIVIDE-AND-CONQUER APPROACH}

In this section we will present an approach to finding an input vector that leads a circuit into a lower (higher) leakage state. We first build a graph model used for finding out clusters of strongly related inputs in a circuit. The purpose of clustering is to enable efficient exhaustive search for the lowest leakage state associated with the inputs in a group. Each group takes its turn to perform exhaustive search until no improvement in the leakage power is observed.

\section{A. Finding Strongly Related Inputs}

We use multi-way graph partitioning tool hMetis [28] to find out the strongly correlated inputs. Herein, primary inputs, latches, and flip-flops will be called inputs to simplify our discussions. In our graph model, we treat an input as a vertex and establish a weighted edge between any two inputs. A weight of an edge between two inputs $P_{i}$ and $P_{j}$ can be defined as:

$$
w_{i, j}=\frac{\alpha C_{i, j}}{\min \left(C_{i}, C_{j}\right)}
$$

where $C_{i}\left(C_{j}\right)$ is the number of gates in the fanout cone rooted at input $P_{i}\left(P_{j}\right)$ and $\alpha$ is a scaling factor. A gate is said to be in the cone $\Lambda_{i}$ rooted at input $P_{i}$ if it can be reached from $P_{i}$ in a topological traversal of the circuit; $C_{i, j}$ is the number of gates both in $\Lambda_{i}$ and $\Lambda_{j}$. The larger an edge weight is, the more strongly the two inputs are correlated. For the example shown in Fig. $3, \Lambda_{1}$ is composed of $g 0, g 1, g 2, g 3, g 5, g 6, g 8$ so that $C_{1}=7$. Similarly, $C_{0}=6$ and $C_{0,1}=4$. Then, $w_{0,1}=66$ given $\alpha=100$ (this is the value used in our experiment). Note that any other weight function can be used if it can reasonably represent the closeness of any two inputs. Once we have the value $w_{i, j}$ for each $\left(P_{i}, P_{j}\right)$ pair, a weighted graph can be created. Note that the weight matrix is symmetric because $C_{i, j}=C_{j, i}$ and $w_{i, j}=w_{j, i}$. It seems that the graph for the above example is quite dense, but we believe that it will be sparser for larger circuits. For a sequential circuit, reset, clear, clock, and other signals that enable normal operations of the circuit should be given proper logic values during standby. Basically, if an input is always fixed at some certain value when a circuit is forced into its standby mode, the input should not be modeled in the weighted graph.

The weight of an edge between $P_{i}$ and $P_{j}$ can be computed by traversing the circuit graph from inputs $P_{i}$ and $P_{j}$ either in depthfirst or breadth-first search. For a circuit with $n$ inputs, we need to compute a weight for each of the $n(n-1) / 2$ edges. This could be an enormous effort for a large circuit. Fortunately, large and highspeed circuits usually have smaller logic depth, which means that the cone of an input can be found quickly. Moreover, we can easily identify whether a cone is contained in another cone without even traversing the circuit graph. Let $L_{i}$ and $L_{j}$ be the set of gates directly connected to inputs $P_{i}$ and $P_{j}$, respectively. If $L_{i} \subseteq L_{j}$ or 


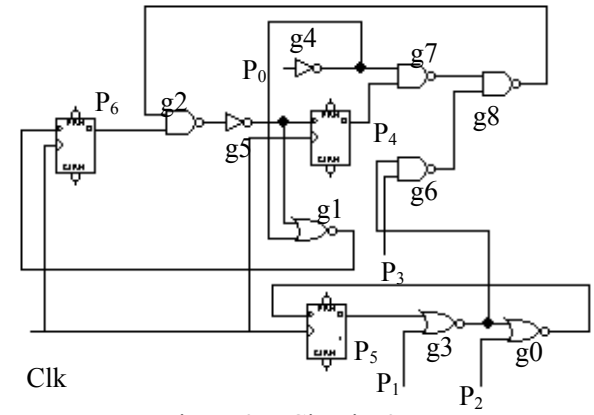

Figure 3. Circuit s27

$L_{j} \subseteq L_{i}$, then $w_{i, j}=\alpha$. For example, in Fig. 3, $L_{1}=L_{5}=\left\{g_{3}\right\}$, thus $w_{1,5}=100$. One step further, let $\Omega_{i}=\left\{\Gamma_{k}|k=1,| L_{i} \mid\right\}$ where $\Gamma_{k}$ is a set of inputs other than $P_{i}$ which can be reached from a gate $g_{k} \in L_{i}$ during backward traversal of the circuit graph from $g_{k}$. If an input $P_{j} \in \bigcap_{k=1}^{\left|L_{i}\right|} \Gamma_{k}$, then $\Lambda_{i} \subseteq \Lambda_{j}$ and thus $w_{i, j}=\alpha$. For example, $L_{6}=\left\{g_{2}\right\}$ and $\Omega_{6}=\left\{\Gamma_{1}\right\}=\left\{\left\{P_{0}, P_{1}, P_{3}, P_{4}, P_{5}\right\}\right\}$, thus $w_{6,0}=w_{6,1}=w_{6,3}=$ $w_{6,4}=w_{6,5}=100$. To identify which input is inspected first, the circuit can be levelized and the inputs directly connecting to a gate with a largest level number are checked first. If the weight of an edge can not be decided in this manner, we have to perform circuit graph traversals starting from the associated inputs to find it. In the worst case, we will take $O\left(n^{2}\right)$ time to compute all the edge weights. However, as we will see later, the weighted graphs for the large circuits can be created within a minute.

Once the weighted graph is created, it can be partitioned into several groups, each of which contains a small number of inputs. In our implementation, the group size ranges from 20 to 25 inputs. In partitioning, the total weight of the edges spanning among groups is minimized such that the inputs in each group form a strongly related cluster. Note that our partitioning approach is different from that presented in $[20,21]$ where logic gates are put into groups, but we put the primary inputs of a circuit into groups.

\section{B. Algorithms}

Once we have done with grouping of inputs, each group takes its turn to perform exhaustive search for a lowest leakage power state. While the inputs in one group are varying their values, the inputs in all other groups remain unchanged. We continue performing exhaustive search until we re-examine a group and see no improvement in the minimum leakage power since the group was last examined. Normally, this process is terminated within a few iterations. Because groups are loosely correlated, the input value changes in one group will not cause logic value changes on too many gates in the cones defined by the inputs in the remaining groups. The algorithm for finding the highest leakage power state can be made similarly. If a circuit has $n$ inputs which are partitioned into $m$ groups, the time complexity for checking all the groups once is $\mathrm{O}\left(m 2^{n / m}\right)$. For a reasonable $m$ value, exhaustive search for a group can be performed very efficiently. Therefore, our algorithm is relatively scalable for the large circuits. A variant of the above algorithm is to process the groups in random order. We find that this variant can be only as good as the fixed order algorithm.

In principle, leakage power needs to be re-computed for every newly applied input vector that causes many gates to switch their logic values. The more inputs change their logic values, the more time will be spent on propagating these changes through a circuit. To minimize the traversing time due to each input application, Gray code rather than binary code is used to generate input vectors for exhaustive search. The Gray code words are generated only once for the largest group and then stored in a table for being used by all the groups. To minimize the memory requirement, only the position of the bit that differs from the previous code word is remembered. We have experienced a significant speedup by just using such a mechanism.

\section{EXPERIMENTAL RESULTS}

ISCAS benchmark circuits are employed to test our approach. To enable leakage power computation, we build a simple cell library based on a $45 \mathrm{~nm}$ technology [27]. The library consists of NANDs, NORs, inverters, and flip-flops. NANDs and NORs have two to four inputs and have $1 \mathrm{X}, 2 \mathrm{X}$, and $4 \mathrm{X}$ driving capabilities. The inverters have $1 \mathrm{X}, 2 \mathrm{X}, 3 \mathrm{X}, 4 \mathrm{X}, 5 \mathrm{X}, 8 \mathrm{X}$, and $16 \mathrm{X}$ driving capabilities. A LUT is created to store the gate and subthreshold leakage for these cells. All the experiments are performed on SUN UltraSPARC 10 workstations with 1G-byte main memory.

TABLE II shows the circuits used for our experiments and the minimum and maximum leakages obtained with $100 \mathrm{~K}$ random vectors, our approach, simulated annealing (SA), and genetic algorithm (GA). ISCAS85 (Cxxx) circuits and ISCAS89 (Sxxx) circuits are tested. The circuits having less than 30 inputs are not shown in the table. In SA approach, the perturbation function generates next feasible solution by inverting the logic value of a randomly selected input. Total leakage current is used as the objective function. In GA approach, the inputs are simply treated as chromosomes and leakage current is used as the fitness value. A new population consists of $60 \%$ of the members from crossover, $10 \%$ from mutation, and $30 \%$ from its ancestors.

All the leakage data in the table are normalized to the minimum leakage obtained with $100 \mathrm{~K}$ random vectors. We find that our algorithm can achieve up to $11 \%$ lower leakage than the lowest and $20 \%$ higher leakage than the highest respectively attainable with $100 \mathrm{~K}$ random vectors for large circuits. Our algorithm excels in obtaining larger maximum leakage for all circuits and smaller minimum leakage for large ISCAS89 circuits, but it does not work well for ISCAS85 circuits in finding smaller minimum leakage. The reason why our algorithm does not work for ISCAS85 circuits is that these circuits normally have larger edge density. This means that the inputs of these circuits are highly correlated so that the groups of inputs found by our algorithm can not be independently set to lower the total leakage. This phenomenon should have also occurred for finding maximum leakage. However, it is not clear why this does not happen. Edge density is a ratio of the number of weighted edges to $n(n-1) / 2$, where $n$ is the number of inputs.

The run time of all the approaches are also shown in Table II. Our approach is significantly faster than others. We let SA and GA approaches run for an extended long time especially for larger ISCAS89 circuits to see whether they are capable of beating our approach. Comparison of the results for ISCAS89 circuits against other approaches depicted in the introduction can not be made because none of these approaches reports data for these circuits.

We also perform many experiments to see how sensitive our approach is to the perturbation incurred by varying the number of parts and the imbalance factor used for graph partitioning. We find that the variation in the percentage of leakage improvement is at most $3 \%$. We have also applied our approach to the case where pin re-ordering [26] is used to lower gate leakage. Compared to the results obtained with $100 \mathrm{~K}$ random vectors, our algorithm also demonstrates its viability in obtaining better minimum/maximum leakage for ISCAS89 circuits. 
TABLE II. EXPERIMENTAL RESULTS

\begin{tabular}{|c|c|c|c|c|c|c|c|c|c|c|c|c|c|c|c|c|c|c|c|}
\hline \multirow{3}{*}{ circuits } & \multicolumn{4}{|c|}{ Circuit Characteristics } & \multicolumn{8}{|c|}{ Leakage Current } & \multicolumn{7}{|c|}{ Runt Time(sec) } \\
\hline & \multirow{2}{*}{$\begin{array}{l}\text { \# of } \\
\text { inputs }\end{array}$} & \multirow{2}{*}{$\begin{array}{l}\text { \# of } \\
\text { cells }\end{array}$} & \multirow{2}{*}{$\begin{array}{c}\text { longest } \\
\text { path } \\
\text { length }\end{array}$} & \multirow{2}{*}{$\begin{array}{l}\text { Edge } \\
\text { density }\end{array}$} & \multicolumn{2}{|c|}{$\begin{array}{c}100 \mathrm{~K} \\
\text { patterns }\end{array}$} & \multicolumn{2}{|c|}{ GA } & \multicolumn{2}{|c|}{ SA } & \multicolumn{2}{|c|}{$\begin{array}{c}\text { Our } \\
\text { Approach }\end{array}$} & \multirow{2}{*}{$\begin{array}{c}100 \mathrm{~K} \\
\text { patterns }\end{array}$} & \multicolumn{2}{|c|}{ GA } & \multicolumn{2}{|c|}{ SA } & \multicolumn{2}{|c|}{$\begin{array}{c}\text { Our } \\
\text { Approach }\end{array}$} \\
\hline & & & & & Min & Max & Min & Max & Min & Max & Min & Max & & Min & Max & Min & Max & Min & Max \\
\hline $\mathrm{C} 432$ & 36 & 299 & 18 & 0.94 & 1 & 1.28 & 0.97 & 1.33 & 0.97 & 1.33 & 1.07 & 1.63 & 438 & 9 & 12 & 4 & 3 & 1 & 2 \\
\hline C1355 & 41 & 475 & 26 & 0.95 & 1 & 1.32 & 0.97 & 1.4 & 0.98 & 1.41 & 1.36 & 1.77 & 1026 & 15 & 25 & 18 & 6 & 0 & 0 \\
\hline $\mathrm{C} 2670$ & 233 & 849 & 21 & 0.34 & 1 & 1.4 & 0.88 & 1.54 & 0.9 & 1.52 & 1.11 & 1.78 & 2929 & 1194 & 860 & 92 & 106 & 43 & 43 \\
\hline C 3540 & 50 & 1207 & 40 & 0.96 & 1 & 1.18 & 0.97 & 1.21 & 0.97 & 1.21 & 1.02 & 1.44 & 3855 & 74 & 70 & 22 & 24 & 4 & 4 \\
\hline C5315 & 178 & 1484 & 39 & 0.40 & 1 & 1.24 & 0.89 & 1.35 & 0.91 & 1.33 & 1.18 & 1.56 & 5577 & 1302 & 1979 & 364 & 200 & 11 & 15 \\
\hline C6288 & 32 & 2385 & 88 & 0.94 & 1 & 1.13 & 0.98 & 1.18 & 0.97 & 1.18 & 0.99 & 1.3 & 6740 & 77 & 101 & 63 & 88 & 13 & 27 \\
\hline C7552 & 207 & 1922 & 42 & 0.92 & 1 & 1.16 & 0.94 & 1.23 & 0.94 & 1.22 & 1.12 & 1.36 & 7039 & 2232 & 1694 & 498 & 597 & 62 & 89 \\
\hline S838 & 67 & 349 & 44 & 0.97 & 1 & 1.24 & 0.92 & 1.31 & 0.92 & 1.31 & 1.11 & 1.67 & 98 & 34 & 33 & 15 & 19 & 2 & 2 \\
\hline S1423 & 92 & 558 & 64 & 0.67 & 1 & 1.19 & 0.95 & 1.28 & 0.95 & 1.26 & 0.94 & 1.26 & 155 & 95 & 115 & 38 & 24 & 6 & 7 \\
\hline S9234 & 182 & 1071 & 22 & 0.32 & 1 & 1.12 & 0.93 & 1.22 & 0.93 & 1.21 & 0.99 & 1.28 & 467 & 1025 & 962 & 116 & 82 & 13 & 14 \\
\hline S5378 & 200 & 1230 & 18 & 0.24 & 1 & 1.06 & 0.98 & 1.09 & 0.99 & 1.09 & 0.99 & 1.16 & 419 & 415 & 440 & 102 & 57 & 10 & 11 \\
\hline S13207 & 680 & 2564 & 28 & 0.11 & 1 & 1.08 & 0.92 & 1.19 & 0.92 & 1.16 & 0.93 & 1.22 & 1864 & 35324 & 47592 & 3109 & 1880 & 38 & 36 \\
\hline $\mathrm{S} 15850$ & 578 & 3239 & 41 & 0.21 & 1 & 1.08 & 0.92 & 1.19 & 0.93 & 1.18 & 0.95 & 1.22 & 1883 & 31021 & 39773 & 3531 & 1707 & 36 & 37 \\
\hline S35932 & 1764 & 8950 & 13 & 0.01 & 1 & 1.11 & 0.96 & 1.14 & 0.93 & 1.16 & 0.89 & 1.15 & 18362 & 86400 & 86400 & 73219 & 44929 & 5223 & 386 \\
\hline S38584 & 1314 & 9356 & 28 & 0.05 & 1 & 1.08 & 0.95 & 1.15 & 0.94 & 1.17 & 0.92 & 1.2 & 13778 & 86400 & 86400 & 38282 & 56943 & 106 & 127 \\
\hline S38417 & 1593 & 9687 & 30 & 0.04 & 1 & 1.06 & 0.93 & 1.13 & 0.91 & 1.15 & 0.89 & 1.19 & 14426 & 86400 & 86400 & 27442 & 35058 & 180 & 199 \\
\hline
\end{tabular}

\section{CONCLUSIONS}

We have proposed an approach to estimating the minimum or maximum leakage for large CMOS circuits. This approach can efficiently find out better vectors that lead up to $11 \%$ lower leakage than the lowest and up to $20 \%$ larger leakage than the highest respectively attainable with $100 \mathrm{~K}$ random vectors for the large ISCAS89 benchmarks. It is simple for implementation. Because of its divide-and-conquer nature, we believe that it will be viable for even larger circuits. However, for ISCAS 85 circuits with large edge density, other approaches should be employed.

\section{REFERENCES}

[1] B. Doyle et al, "Transistor Elements for 30nm Physical Gate Lengths and Beyond," Intel Tech. Journal, Vol.6,Issue 2, May 2002, pp.42-54.

[2] D. Eckerbert and P. Larsson-Edefors, "Cycle-True Leakage Current Modeling for CMOS Gates," ISCAS, Vol. 5, 2001, pp. 507-510.

[3] A. Keshavarzi et al, "Technology Scaling Behavior of Optimum Reverse Body Bias for Standby Leakage Power Reduction in CMOS IC's," ISLPED, 1999, pp. 252-254.

[4] Y.-S. Lin et al, "Leakage Scaling in Deep Submicron CMOS for SoC," IEEE Trans. on Electron Devices, Vol. 49, No. 6, June 2002, pp. 1034-1041.

[5] K. Roy, S. Mukhopadhyay, and H. Mahmoodi-Meimand, "Leakage Current Mechanisms and Leakage Reduction Techniques in DeepSubmicrometer CMOS Circuits," Proc. of the IEEE, Vol. 91, No. 2, Feb. 2003, pp. 305-327.

[6] Z. Chen, C. Diaz, J. D. Plummer, M. Cao, and W. Greene, "0.18um Dual $\mathrm{V}_{\mathrm{t}}$ MOSFET Process and Energy-Delay Measurement," IEDM, 1996, pp. 851-854.

[7] L. Wei et al, " Design and Optimization of Dual-Threshold Circuits for Low-Voltage Power Applications," IEEE Trans. on VLSI Systems, Vol. 7, No. 1, March 1999, pp. 16-24.

[8] T. Kawahara, Y. Kawajiri, G. Kitsukawa, T. Kure, and M. Aoki, "Subthreshold Current Reduction for Decoded-Driver by Self-reverse Biasing," IEEE JISSC, Vol. 28, No.11, Nov 1993, pp. 1136-1144.

[9] K. Seta, H. Hara, T. Kuroda, M. Kakumu, and T. Sakurai, " $50 \%$ Active-Power Saving without Speed Degradation Using Standby Power Reduction (SPR) Circuit," IEEE ISSCC, 1995, pp. 318-319.

[10] T. Douseki, S. Shigematsu, J. Yamada, M. Harada, H. Inokawa, and T. Tsuchiya, "A 0.5-V MTCMOS/SIMOX Logic Gate,” IEEE JSSC, Vol. 32, No. 10, 1997, pp. 1604-1609.

[11] Z. Chen, M. Johnson, L. Wei, and K. Roy, "Estimation of Standby Leakage Power in CMOS Circuits Considering Accurate Modeling of Transistor Stacks," ISLPED, 1998, pp. 239-243.
[12] M. C. Johnson, D. Somasekhar, L.-Y. Chiou, and K. Roy, "Leakage Control With Efficient Use of Transistor Stacks in Single Threshold CMOS," IEEE Trans. on VLSI Systems, Vol. 10, No. 1, Feb 2002, pp. $442-445$.

[13] N. Hanchate and N. Ranganathan, "LECTOR: A Technique for Leakage Reduction in CMOS Circuits," IEEE Trans. On VLSI Systems, Vol. 12, No. 2, pp. 196-205, Feb. 2004.

[14] J. P. Halter and F. N. Najm, "A Gate-Level Leakage Power Reduction Method for Ultra-Low-Power CMOS Circuits," CICC, 1997, pp. 475-78.

[15] M. C. Johnson, D. Somasekhar, and K. Roy, "Models and Algorithms for Bounds on Leakage in CMOS Circuits," IEEE Trans. on CAD, Vol. 18, No. 6, June 1998, pp. 714-725.

[16] S.R. Naidu and E.T.A.F. Jacobs, "Minimizing Stand-by Leakage Power in Static CMOS Circuits," DATE, 2001, pp. 370-376.

[17] F. A. Aloul et al, "Robust SAT-Based Search Algorithm for Leakage Power Reduction," International Workshop on Power and Timing Modeling, Optimization and Simulation, 2002.

[18] A. Abdollahi and F. Fallah, and M. Pedram, "Leakage Current Reduction in CMOS VLSI Circuits by Input Vector Control," IEEE Trans. on VLSI Systems, Vol. 12, No. 2, Feb. 2004, pp. 140-154.

[19] S. Bobba and I. N. Hajj, "Maximum Leakage power Estimation for CMOS Circuits;" IEEE Alessandro Volta Memorial Workshop on Low-Power Design, Mar. 1999, pp. 116-124.

[20] A. Ferre and J. Figueras, "Leakage Power Bounds in CMOS Digital Techniques," IEEE Trans. On CAD,Vol.21, No.6, June 2002, pp. 731-738

[21] Kaviraj Chopra and Sarma B. K. Vrudhula, "Implicit Pseudo Boolean Enumeration Algorithms for Input Vector Control," ACM/IEEE DAC, 2004, pp.767-772.

[22] F. Hamzaoglu and M. Stan, "Circuit-Level Techniques to Control Gate Leakage for Sub-100 nm CMOS," ISPLD, 2002, pp. 60-63.

[23] M. Drazdziulis and P. Larsson-Edefors, "A Gate Leakage Reduction Strategy for Future CMOS Circuits,” ESSCIRC, 2003, pp. 317 - 320.

[24] R. S. Guindi and F. N. Najm, "Design Techniques for Gate-Leakage Reduction in CMOS Circuits," ISQED, 2003.

[25] S. Mukhopadhyay et al, "Gate Leakage Reduction for Scaled Devices Using Transistor Stacking," IEEE Trans. on VLSI Systems, Vol. 11, No. 4, Aug. 2003, pp. 716-730.

[26] D. Lee, D. Blaauw, and D. Sylvester, "Gate Oxide Leakage Current Analysis and Reduction for VLSI Circuits," IEEE Trans. on VLSI Systems, Vol. 12, No. 2, Feb. 2004, pp. 155-166.

[27] K. M. Cao et al, "BSIM4 Gate Leakage Model Including SourceDrain Partition," IEDM, 2000, pp. 35.3.1-35.3.4.

[28] http://www.cs.umn.edu/metis. 